\title{
LOWER AND UPPER BOUNDS TO THE ULTIMATE LOADS OF BUCKLED REDUNDANT TRUSSES*
}

\author{
BY \\ E. F. MASUR \\ Illinois Institute of Technology
}

Synopsis. In the buckled state, statically indeterminate, rigid-jointed trusses support loads which are generally in excess of those corresponding to initial instability. As buckling proceeds, the loads usually approach limiting values, called "ultimate loads". Two theorems are derived establishing lower and upper bounds to the ultimate loads. Elastic behavior is assumed throughout.

Introduction. If the rigid joints of a truss are subjected to a set of external loads, whose values are fixed except for a common multiplier $\lambda_{0}$, the equations of equilibrium of the $i$-th joint are of the type ${ }^{1}$

$$
\begin{aligned}
& \sum_{i} S_{i i}\left\{\begin{array}{c}
\cos \phi_{i j} \\
\sin \phi_{i i}
\end{array}\right\}+\lambda_{0}\left\{\begin{array}{l}
X_{i} \\
Y_{i}
\end{array}\right\}=0 \quad(i=1,2, \cdots n) \\
& \sum_{i} M_{i i}=0 \quad(i=1,2, \cdots n)
\end{aligned}
$$

where $S_{i j}$ and $M_{i i}$ are, respectively, the axial force and bending moment in bar $(i, j)$ at joint $i, \phi_{i i}$ is the inclination of bar $(i, j), X_{i}$ and $Y_{i}$ are external force components, and where the summation extends over all the bars adjoining $i$. If now all bars are composed of an elastic material and the elementary theory of beams in bending under axial forces is assumed to hold, the joint moments $M_{i i}$ can be expressed as linear functions of the joint rotations $\theta_{i}$ [2]. Substitution of these expressions into Eqs. (1b) leads to a set of linear, homogeneous equations in the joint rotations of the type

$$
\sum_{i=1}^{n} a_{i j} \theta_{i}=0, \quad(i=1,2, \cdots n)
$$

where the $a_{i j}$ form a symmetric matrix whose components are transcendental functions of the physical characteristics of the bars, and of the axial forces. The equilibrium of the structure is stable only if the matrix is positive definite; a necessary condition of neutral equilibrium is given by

$$
f\left(\lambda_{0}\right) \equiv\left|a_{i j}\right|=0 .
$$

The lowest positive real root of Eq. (3) is the desired "critical" multiplier $\lambda_{0}$ at which buckling occurs.

By definition, the bar forces $S_{i j}$ are uniquely determined by Eqs. (1a) for given $\lambda_{0}$ in a statically determinate truss. If, on the other hand, the truss be of $m$-th degree of redundancy relative to its axial force distribution, the most general expression for the bar forces is of the form

$$
S_{i i}=\sum_{\xi=0}^{m} \lambda_{\xi} S_{i i}^{(\xi)}
$$

${ }^{*}$ Received Nov. 21, 1952.

'The effect of the linear displacements is ignored in Eqs. (1) and (2). This is in conformity with the experimental results given in Ref. [1] (see the bibliography at the end of the paper). 
in Eqs. (4), the force systems $S_{i j}^{(r)}$ are restricted by the set of equilibrium equations

$$
\sum_{i} S_{i j}^{(r)}\left\{\begin{array}{c}
\cos \phi_{i i} \\
\sin \phi_{i j}
\end{array}\right\}+\delta_{0 r}\left\{\begin{array}{c}
X_{i} \\
Y_{i}
\end{array}\right\}=0, \quad(r=0,1, \cdots m ; i=1,2, \cdots n)
$$

where $\delta_{p a}$ is the Kronecker Delta.

It is convenient to restrict the force systems $S_{i i}^{(r)}$ further by the requirement (which can always be fulfilled) that

$$
\begin{aligned}
\sum_{k} \frac{S_{k}^{(0)} S_{k}^{(r)} L_{k}}{E_{k} A_{k}}=p \delta_{0 r}, & (r=0,1,2, \cdots m) \\
\sum_{k} \frac{S_{k}^{(r)} S_{k}^{(s)} L_{k}}{E_{k} A_{k}}=\delta_{r s}, & (r, s=1,2, \cdots m)
\end{aligned}
$$

where the summation extends over all the members of the truss. ${ }^{2}$ Eqs. (6a) indicate that the force system $S_{i}^{(0)}$ is the actual force system, i.e. the one corresponding to minimum strain energy, in the unprestressed, unbuckled truss for $\lambda_{0}=1$.

The axial forces in the buckled state are further restricted by the "compatibility condition" that

$$
\delta L_{k}=\left(u_{i}-u_{i}\right) \cos \phi_{i j}+\left(v_{i}-v_{i}\right) \sin \phi_{i j},
$$

in which $\delta L_{k}$ is the change in the distance between joints $i$ and $j$ relative to the unloaded state and $(u, v)$ are the cartesian components of the linear displacement vectors of the joints. If both sides of Eqs. (7) be multiplied by $S_{k}^{(r)}(r=1,2, \cdots m)$ and summed up over all the members of the truss, we are led, after rearranging the summations and in view of Eqs. (5), to the system of "virtual work" equations

$$
\sum_{k} S_{k}^{(r)} \delta L_{k}=0 . \quad(r=1,2, \cdots m)
$$

For the sake of generality it will be assumed that the truss has been prestressed, with the initial force system expressed by

$$
S_{k}^{*}=\sum_{\xi=1}^{m} \lambda_{\xi}^{*} S_{k}^{(\xi)} .
$$

On the other hand, $\delta L_{k}$ can be written in the form

$$
\delta L_{k}=\frac{\left(S_{k}-S_{k}^{*}\right) L_{k}}{E_{k} A_{k}}-\delta_{k},
$$

where $\delta_{k}$ represents the shortening of the chord length of the $k$-th member corresponding to its curvature in bending. If we substitute Eqs. (10) in (8) and consider Eqs. (6) and (9), we arrive at the following set of equations for any bent state:

$$
\lambda_{r}-\lambda_{r}^{*}=\sum_{k} S_{k}^{(r)} \delta_{k} . \quad(r=1,2, \cdots m)
$$

It has been shown elsewhere [3] that, for an actual buckling mode,

$$
\sum_{k} S_{k}^{(r)} \delta_{k}=\frac{1}{2} \sum_{i} \sum_{i} a_{i j, r} \theta_{i} \theta_{i}=\mu f_{, r} \quad(r=0,1,2, \cdots m)
$$

${ }^{2} S_{k}, L_{k}, E_{k}, A_{k}$ are the axial force, length, modulus of elasticity and cross-sectional area, respectively, of the $k$-th bar. 
in which a subscript, preceded by a comma, designates the partial derivative with respect to the corresponding parameter $\lambda$, or $f_{, r} \equiv \partial f / \partial \lambda_{r}$. Thus, during buckling,

$$
\lambda_{r}-\lambda_{r}^{*}=\mu f_{, r}, \quad(r=1,2, \cdots m)
$$

where $\mu>0$ is a measure of the extent to which buckling has proceeded and is expressed by

$$
\mu=\frac{1}{2} \frac{\sum_{i=1}^{n} \theta_{i}^{2}}{\sum_{i=1}^{n} A_{i i}},
$$

in which $A_{i i}$ is the cofactor of $a_{i i}$ in Eq. (3). It follows immediately from Eqs. (3) and (12) that, as buckling commences, the load $\lambda_{0}$ will generally increase, and never decrease. Considering all parameters $\lambda_{r}(r=0,1,2, \cdots m)$ to be functions of $\mu$, Eq. (3) can be written in the form

$$
F(\mu) \equiv f\left[\lambda_{0}(\mu), \lambda_{1}(\mu), \cdots \lambda_{m}(\mu)\right] \equiv 0
$$

for all values of $\mu$. Differentiating Eq. (13) with respect to $\mu$, we obtain the identity

$$
\frac{d F}{d \mu} \equiv f_{, 0} \frac{d \lambda_{0}}{d \mu}+\sum_{\xi=1}^{m} f_{. \xi} \frac{d \lambda_{\xi}}{d \mu} \equiv 0
$$

On the other hand, by dividing Eq. (12a) by $\mu$ and letting $\mu$ approach zero, we are led to

$$
\frac{d \lambda_{r}}{d \mu}(\mu=0)=f_{. r}(\mu=0) \quad(r=1,2, \cdots m)
$$

It follows, from Eqs. (14) and (15), that as the truss is on the verge of buckling

$$
\text { - } \frac{d \lambda_{0}}{d \mu}=-\left(f_{.0}\right)^{-1} \sum_{\xi=1}^{m}\left(f_{, \xi}\right)^{2},
$$

where all the terms in Eq. (16) are to be evaluated at $\mu=0$.

It can be shown that $f_{, 0}$ is negative. In fact, if $\lambda_{0}^{1}$ be the smallest positive buckling parameter, i.e. the one corresponding to neutral equilibrium, the positive definiteness of the matrix $\left[a_{i j}\right]$ implies $f\left(\lambda_{0}\right)>0$ for $\lambda_{0}<\lambda_{0}^{1}$. Since, by Eq. (3), $f\left(\lambda_{0}^{1}\right)=0$, and if multiple roots be excluded, it follows that

$$
f_{.0}\left(\lambda_{0}=\lambda_{0}^{1}\right)<0 .
$$

An inspection of Eqs. (16) and (17) shows that

$$
\frac{d \lambda_{0}}{d \mu}(\mu=0) \geq 0,
$$

where the equality sign applies only to the special case

$$
f_{, r}\left(\lambda_{r}=\lambda_{r}^{*}\right)=0 . \quad(r=1,2, \cdots m)
$$

It follows further from Eqs. (12) that for increasing $\mu$, and provided the $\lambda_{r}$ all stay

\footnotetext{
${ }^{3}$ Actually, this relationship applies to all values of $\mu$, as can be demonstrated by means of energy considerations.
} 
finite, ${ }^{4}$ the external and internal forces approach a state governed by the equations

$$
\begin{aligned}
& f\left(\lambda_{0}^{u}, \lambda_{1}^{u}, \cdots \lambda_{m}^{u}\right)=0, \\
& f_{. r}\left(\lambda_{0}^{u}, \lambda_{1}^{u}, \cdots \lambda_{m}^{u}\right)=0, \quad(r=1,2, \cdots m)
\end{aligned}
$$

in which the superscript designates limiting, or "ultimate" values of the parameters, with $\lambda_{0}^{u}$ referred to as the "ultimate load".

The determination of $\lambda_{0}^{u}$ is of interest to the engineer since it represents the ultimate carrying capacity of the truss. Since, however, the solution of Eqs. (19) presents formidable numerical obstacles, it is easier to estimate the value of $\lambda_{0}^{u}$ by means of two theorems establishing lower and upper bounds to the ultimate load.

A stability criterion. Before proceeding to these two theorems, it is convenient to establish a stability criterion for the unbuckled truss, which is to be used in the later proof. This criterion is based on the assumption that, for a given system of external forces identified by the load parameter $\lambda_{0}$, the potential energy corresponding to the unbuckled state is less than that of any neighboring, "geometrically consistent" bent state if the equilibrium of the truss is to be stable, or

$$
V^{*}<V \text {. }
$$

In what follows, a geometrically consistent bent state is defined as one in which the deflection curves of the bars are of sufficient degree of smoothness to make the discussion meaningful, and satisfy the geometric boundary conditions of continuity at the joints. Geometric consistency also implies the satisfaction of Eqs. (7) and (10); it is finally assumed that the force equations (4), (5), and (6), and therefore also Eqs. (8) and (11a), are valid.

With these definitions, the potential energy

$$
V=U-W=\frac{1}{2} \sum_{k} \frac{S_{k}^{2} L_{k}}{E_{k} A_{k}}+U_{B}-\lambda_{0} \sum_{i}\left(X_{i} u_{i}+Y_{i} v_{i}\right),
$$

in which $U_{B}$ is the strain energy associated with the bending of the truss bars, can be expressed by Eqs. (4), (5), (6) and (7) in the form

$$
V=\frac{1}{2} p \lambda_{0}^{2}+\frac{1}{2} \sum_{\xi=1}^{m} \lambda_{\xi}^{2}+U_{B}-\sum_{k} S_{k} \delta L_{k} ;
$$

in view of Eqs. (8), (9), and (10), this reduces to

$$
V=-\frac{1}{2} p \lambda_{0}^{2}+\frac{1}{2} \sum_{\xi=1}^{m} \lambda_{\xi}^{2}+U_{B}+\lambda_{0} \sum_{k} S_{k}^{(0)} \delta_{k} .
$$

Similarly, the potential energy in the unbent state is given by

$$
V^{*}=-\frac{1}{2} p \lambda_{0}^{2}+\frac{1}{2} \sum_{\xi=1}^{m} \lambda_{\xi}^{*^{2}}
$$

Thus, criterion (20) now takes the form

$$
V-V^{*}=\frac{1}{2} \sum_{\xi=1}^{m}\left(\lambda_{\xi}-\lambda_{\xi}^{*}\right)\left(\lambda_{\xi}+\lambda_{\xi}^{*}\right)+L_{B}+\lambda_{0} \sum S_{k}^{(0)} \delta_{k} \geq 0
$$

${ }^{4}$ For a discussion of this question see Ref. [3]. 
which, by Eqs. (11a) and by considering only states in the neighborhood of the unbuckled state, is finally expressed as

$$
V-V^{*}=\sum_{k} S_{k} \delta_{k}+U_{B} \geq 0,
$$

where the equality sign applies to the case of neutral equilibrium, with the bent state represented by the first buckling mode. The stability criterion (21) can also be demonstrated directly. In fact, if the arbitrary set of deflection curves $y_{k}(x)$ be developed in a Fourier series

$$
y_{k}(x)=\sum_{\tau=1}^{\infty} c_{\tau} \eta_{k}^{\tau}(x) .
$$

in which $\eta_{k}^{i}(x)$ represents the normalized buckling mode associated with the positive load parameter $\lambda_{0}^{i}$, it can be shown, by a series of partial integrations and by virtue of the orthogonality of the normal modes, that

$$
\sum_{k} S_{k} \delta_{k}+U_{B}=\sum_{\tau=1}^{\infty} c_{\tau}^{2}\left(\lambda_{0}^{\tau}-\lambda_{0}\right) .
$$

The right side of this equation is positive definite if $\lambda_{0}<\lambda_{0}^{1}$, i.e. when the load parameter is smaller than the smallest buckling load.

Lower bounds to the ultimate load. After these preliminary remarks, we now proceed to state the first theorem. In what follows, we shall call a load parameter $\lambda_{0}>0$ "critical" if there exists a set of prestressing parameters $\lambda_{r}^{*}(r=1,2 \cdots m)$ such that the truss is in neutral equilibrium with its force system defined by $\left(\lambda_{0}, \lambda_{1}^{*}, \cdots \lambda_{m}^{*}\right)$. With this definition, which is analogous to that of a "statically admissible multiplier" in rigid-plastic limit design [4], we state

Theorem I: The ultimate load is the largest of all critical loads.

From this theorem we deduce the following

CoRoLlary: If an arbitrary internal force system, and an external load system identified by $\lambda_{0}^{\prime}$, satisfy the force equations of equilibrium, and if the truss, with its forces so defined, is in stable equilibrium, then

$$
\lambda_{0}^{u}>\lambda_{0}^{\prime} \text {. }
$$

It is clear that this corollary establishes an easily calculable lower bound to the ultimate load since the stability of a truss for a given force system can be determined by a number of stability criteria $[5,6]$.

PRoof: It can readily be verified that if the prestressing parameters be so selected that $\lambda_{r}^{*}=\lambda_{r}^{u}(r=1,2: \cdots m)$, Eqs. (3) and (12a) are identically satisfied by

$$
\lambda_{r} \equiv \lambda_{r}^{u} \quad(r=0,1,2, \cdots m)
$$

for all values of $\mu$. In other words, in this "special case", the truss buckles similarly to a statically determinate truss under constant external loads and internal forces. ${ }^{5}$

${ }^{5}$ It has been shown previously [7] that this case corresponds to a stationary value of the buckling load. See also Eq. (18). 
Inspection of Eq. (11a) shows that the buckling mode of the special case is such as to satisfy

$$
\sum_{k} S_{k}^{(r)} \delta_{k}=0 . \quad(r=1,2, \cdots m)
$$

If now the stability criterion (21) be applied to a truss in stable equilibrium under an external load $\lambda_{0}^{\prime}$, and if the arbitrary deflection curves be so selected as to coincide with the buckling mode of the "special case", it follows from Eqs. (23) that

$$
V-V^{*}=\lambda_{0}^{\prime} \sum_{k} S_{k}^{(0)} \delta_{k}+U_{B}>0 .
$$

Since, also by Eqs. (21) and (23),

$$
\lambda_{0}^{u} \sum_{k} S_{k}^{(0)} \delta_{k}+U_{B}=0,
$$

and since further $U_{B}$ is positive definite, it follows that

$$
\lambda_{0}^{u}>\lambda_{0}^{\prime} .
$$

Upper bounds to the ultimate load. Before proceeding to the discussion of upper bounds to the ultimate load, it is useful to state the following

Lemma I: For a given set of prestressing parameters, the value of the critical load of a truss is not reduced by increasing the moment of inertia of a bar. ${ }^{6}$

Proof: As stated before, a truss is in stable equilibrium only if the matrix of the coefficients $a_{i j}$ is positive definite, or

$$
Q=\frac{1}{2} \sum_{i} \sum_{i} a_{i j} \theta_{i} \theta_{i} \geq 0
$$

where the equality sign applies only to the trivial case $\theta_{i}=0(i=1,2 \cdots n)$. By rearranging the terms in the double sum, this can be expressed [8] in the form

$$
Q=\frac{1}{8} \sum_{k} S_{k} L_{k}\left[\left(\epsilon_{k} \operatorname{coth} \epsilon_{k}-1\right)^{-1}\left(\theta_{k}+\theta_{k}^{\prime}\right)^{2}+\left(\epsilon_{k}\right)^{-1}\left(\theta_{k}-\theta_{k}^{\prime}\right)^{2} \operatorname{coth} \epsilon_{k}\right],
$$

where $\epsilon_{k}$ is defined by

$$
\epsilon_{k}^{2}=\frac{S_{k} L_{k}^{2}}{4 E_{k} I_{k}}>-\pi^{2}
$$

and where $\theta_{k}$ and $\theta_{k}^{\prime}$ are, respectively, the rotations of the two joints connected by the $k$-th bar of moment of inertia $I_{k}$.

Let us now assume that a truss $T$ is in stable equilibrium for a given set of internal and external forces, and consider the equilibrium, under the same force system, of a truss $T^{\prime}$ which is identical with $T$ as to its geometric and elastic properties, except that the moment of inertia of its $m$-th bar has been increased, i.e.

$$
I_{m}^{\prime}>I_{m} \text {. }
$$

If this bar be a tension member, it follows from Eq. (25b) that

$$
\left(\epsilon_{m}^{\prime}\right)^{2}<\epsilon_{m}^{2}
$$

'This lemma, which has immediate physical appeal, is analogous to a similar principle of Rayleigh dealing with the natural frequencies of vibration of elastic systems. 
and, by inspection of Eq. (25a), that the value of the $m$-th term in the series has not decreased. The same can readily be demonstrated for compression members, which correspond to imaginary values of $\epsilon$, and for unstressed members. Consequently, for any given set of joint rotations,

$$
Q^{\prime} \geq Q ;
$$

in view of Eq. (24), the truss $T^{\prime}$ is therefore also in stable equilibrium.

As a next step, it is easy to establish the following

LEMma II: The value of the ultimate load of a truss is not lowered by increasing the moment of inertia of a bar.

Proof: This is a direct consequence of the previous lemma and of Theorem I. In fact, if the prestressing parameters $\lambda_{r}^{*}(r=1,2 \cdots m)$ be so selected as to make the critical load $\lambda_{0}$ equal to the ultimate load $\lambda_{0}^{u}$ for truss $T$, it follows from Lemma $I$ that, for the same $\lambda_{r}^{*}$, the critical load $\lambda_{0}^{\prime}$ of truss $T^{\prime}$ satisfies the relationship

$$
\lambda_{0}^{\prime} \geq \lambda_{0}=\lambda_{0}^{u} \text {. }
$$

On the other hand, it follows from Theorem I that the ultimate load $\lambda_{0}^{\prime \prime}$ of truss $T^{\prime}$ is the largest of all critical loads of $T^{\prime \prime}$, or

$$
\lambda_{0}^{\prime u} \geq \lambda_{0}^{\prime}
$$

which, in view of the foregoing, implies

$$
\lambda_{0}^{\prime u} \geq \lambda_{0}^{u} .
$$

An upper bound to the ultimate load can now be established by means of the fact that a truss of $m$-th degree of redundancy can be converted into a rigid-link mechanism of one degree of freedom by considering it pin-jointed and by removing $l$ members, where $l \leq m+1$. In general, it will be possible ${ }^{7}$ to select these $l$ members in such a way that the mechanism so created is capable of joint velocities corresponding to a shortening of all the eliminated bars.

If now these $l$ members be assigned compressive forces

$$
S_{k}=-4 \pi^{2} E_{k} I_{k}\left(L_{k}\right)^{-2} \quad(k=1,2, \cdots l)
$$

and if the remaining bar forces be so chosen as to satisfy the force equations of equilibrium (2a) for $\lambda_{0}=\lambda_{0}^{\prime \prime}>0$, then, by

Theorem II:

$$
\lambda_{0}^{u} \leq \lambda_{0}^{\prime \prime} .
$$

In other words, the load parameter found by the method described above represents an upper bound to the ultimate load.

The proof of this follows from the second lemma. In fact, since Eq. (27) defines the buckling load of a column which is fixed at both ends, it is apparent that $\lambda_{0}^{\prime \prime}$ can be interpreted as the ultimate load of a truss which is identical with the truss under

\footnotetext{
'Otherwise, the truss does not exhibit an ultimate load, i.e., the load increases indefinitely during buckling.
} 
consideration except that all bars other than the selected $l$ bars described above have infinite moment of inertia. Thus the second lemma implies the relationship (28).

It should be pointed out that the practical usefulness of the upper bound defined by Theorem II is sharply limited. A more detailed discussion of this question can be found elsewhere [3]; suffice it to state here that the theorem is much less broad in scope than a similar one establishing upper bounds to the collapse load of a plastic-rigid frame. [4] This is due to the fact that it has not been possible to construct an infinity of readily calculable "kinematically admissible" collapse modes by any simple process analogous to the insertion of a sufficient number of yield hinges. Fortunately, except for the purpose of estimating the degree of accuracy attained, the interest of the engineer is focused on the lower bound only, which can be made to approach the exact value of the collapse load as closely as desired.

Conclusion. Two principles have been derived which establish lower and upper bounds to the ultimate load sustained by a buckled redundant truss. Of these, the lower bound permits an approach to the exact value from below within any desired degree of accuracy.

\section{BibLIOGRAPHY}

1. N. J. Hoff, B. A. Boley, S. V. Nardo, and S. Kaufman, Buckling of rigid-jointed plane trusses, Proc. Separate No. 24, Am. Soc. Civ. Engrs., 6, (1950).

2. B. W. James, Principal effects of axial load on moment-distribution analysis of rigid structures, Techn. Note No. 534, NACA, (1935).

3. E. F. Masur, Post-buckling strength of redundant trusses, Proc. Separate, Am. Soc. Civ. Engrs. (publication pending).

4. H. J. Greenberg and W. Prager, Limit design of beams and frames, Proc. Separate No. 59, Am. Soc. Civ. Engrs., 2, (1951).

5. E. E. Lundquist, Stability of structural members under axial load, Tech. Note No. 617, NACA, (1937).

6. N. J. Hoff, Stable and unstable equilibrium of plane frameworks, J. Aero. Sci. 8, 115 (1941).

7. E. F. Masur, The effect of prestressing on the buckling loads of statically redundant, rigid-jointed trusses, Proc. First U. S. National Congress Appl. Mech., Chicago, Illinois.

8. E. F. Masur, The stability of statically indeterminate, rigid-jointed trusses, Unpublished Thesis, Illinois Institute of Technology, Chicago, Illinois. 